\title{
A beneficial effect of part-list cuing with unrelated words
}

\author{
CATHERINE G. PENNEY \\ Memorial University of Newfoundland, St. John's, Newfoundland, Canada
}

\begin{abstract}
Previous experiments have found no beneficial effect of providing some list items as retrieval cues for other words in the list. This finding contradicts organizational theory, which predicts a positive effect of intralist cues on recall. The hypothesis tested in the present study was that intralist cues would increase recall when the following conditions are met: (1) subjects form integrated subjective units during learning, (2) not all of these units are recalled in the absence of cues, and (3) appropriate retrieval cues are selected to access these units. University students sorted a list of 64 unrelated words into subjective categories and then attempted to recall the words. For cued recall trials, one word from each subjective unit was chosen as a cue. More target words were recalled in cued recall than in free recall. This finding is consistent with organizational theory and indicates that experiments that found no beneficial effects of intralist cues did not employ optimal organization and cuing conditions.
\end{abstract}

Considerable evidence has accumulated in the last 20 years regarding the role of organization in free recall. In a typical experiment on free recall, a subject studies a list of words and then attempts to recall the words in any order. No specific retrieval cues are provided. Performance in this task has been shown to depend either upon development of an organizational schema that incorporates most of the list words, such as a narrative, or upon the association of list words to a previously learned set of retrieval cues, such as the alphabet, a set of peg words, or a sequence of loci. Retrieval of a target item is achieved when that target can be reached through an association link established during the study period to either a list word that has already been recalled or to an item such as a locus, a peg word or letter of the alphabet that is easily recallable.

According to organization theory, list words become organized during learning into chunks or subjective units. Failure to recall at least some of the words is believed to reflect the failure to retrieve entire chunks. When categorized lists of words are studied, subjects often fail to recall entire categories. When provided with the names of the categories (Tulving \& Pearlstone, 1966) or with one item from each category (Wood, 1969), subjects are able to recall more of the remaining instances than when cues are not provided. Beneficial effects of cuing should also be found when lists of unrelated words are used, but this situation is not as clear-cut. Dong and Kintsch (1968) had subjects sort words into subjective categories and give a label for each category. These labels were shown to be effective retrieval cues.

With categorized word lists, list items are effective retrieval cues for other items from the same category; in

Address correspondence to Catherine G. Penney, Psychology Department, Memorial University of Newfoundland, St. John's, Newfoundland A1B 3X9, Canada. contrast, when the list items are unrelated to one another, the presence of list items on the retention test appears to reduce recall relative to an uncued condition (Slamecka, $1968,1969)$. In a series of experiments, Slamecka (1968, 1969) compared recall performance under the typical free recall condition with performance in a condition in which some of the list items were provided as retrieval cues. It would seem that if the list items become organized into chunks or subjective units during learning and if some of these chunks are not retrievable in a free recall test, then providing a portion of the list words should facilitate access to these irretrievable chunks. Recall of the comparable target words should be higher in conditions in which the list words are given as retrieval cues than in free recall conditions. In contrast to the prediction, Slamecka found that recall was actually lower when the cues were provided.

In replications of Slamecka's $(1968,1969)$ studies (see Nickerson, 1984, for a review), the negative effect of the intralist cues was not always found, but there was only one experiment (by Reardon, Polzella, \& Brown, 1975) that showed evidence of the predicted beneficial effect of the cues. Reardon et al. gave subjects a list of 84 words and asked them to sort the words into groups of three "related" words. The retention test then consisted of three conditions. In the group cue condition, two words from each of 12 groups were provided as cue doublets, and in the random cue condition, two words from each of another 12 groups were randomly repaired as cue doublets. For both of these conditions, the remaining words were the target items. For the remaining four word groups created by a subject, none of the list items appeared on the retention test. This left 12 target items in each condition (random cue, group cue, and no cue). Reardon et al. found that the highest proportion of target items was recalled in the group cue condition and the lowest proportion in the uncued condition. 
The present research replicates and extends the work of Reardon et al. (1975) and focuses on the conditions under which a beneficial effect of intralist cues is expected. A beneficial effect of intralist cues has not usually been found with lists of unrelated words, because the conditions that are required for the cues to be effective have not been met. When these conditions are met, as in the study reported here, a beneficial effect of intralist cues is found.

First, in order for an item to be an effective retrieval cue, it must be strongly associated with its target items. In the case of categorized or associated word lists, the association between cue and target has been established before the experiment begins, but with unrelated words, no such preexperimental association exists. The study phase of the experiment must therefore promote the formation of strong associations between the words to be used as retrieval cues and those to be target items.

In Slamecka's $(1968,1969)$ experiments the organization that developed during learning and the selection of retrieval cues may not have been appropriate for a test of intralist cue effectiveness. Suppose that during study a number of list items did become associated in some kind of network or hierarchial organizational schema. In order for intralist cues to be effective in retrieving unrecalled items, these words must be organized into chunks of at least two items. In Slamecka's experiments, it is likely either that unrecalled items were not organized into chunks or that, because the subjects' organizational schemata were unknown to the experimenter, appropriate retrieval cues for the unrecalled chunks were not selected.

The development of chunks of strongly associated words was achieved by Reardon et al. (1975) through the use of the sorting procedure developed by Mandler (1967; Mandler \& Pearlstone, 1966). In the experiments reported here, subjects were instructed to sort a list of 64 unrelated words into groups, so that the items in a group were related in some way. The first word in each group was selected as the retrieval cue. In this way, the words selected as retrieval cues were strongly associated with the other words in the subjective unit and were likely, therefore, to be effective retrieval cues.

A second condition that must be met for intralist cues to be effective is that there must be a number of chunks of at least two words that are not retrieved in a free recall test but are potentially retrievable with an appropriate cue. If the level of learning is very high or the interchunk organization very strong, then there will be no chunks that are unretrievable in a free recall test but potentially retrievable if appropriate cues are given. Mandler's sorting technique is a suitable method for promoting the organization of a reasonably long list of words into chunks of three or four items each. The items within a chunk will be strongly associated, but there is little reason for associations to occur between words in different chunks. It is highly likely, therefore, that there will be entire chunks that are not recalled in a free recall test but that are potentially retrievable.
Two experiments were carried out. In both experiments, subjects first sorted a list of 64 unrelated words into subjective categories under incidental learning conditions. After the subjects had achieved a suitable sorting of the words, both free recall and cued recall tests were administered. In Experiment 1, the free recall test preceded the cued recall test; in Experiment 2, the cued recall came first. The prediction from organizational theory is that in both experiments, recall of target items in the cued recall condition should exceed recall of those items in the free recall condition. Furthermore, if the cues promote recall of subjective units, the improved recall in the cued condition should be reflected in the number of categories recalled rather than in the size of the categories.

\section{METHOD}

\section{Subjects}

Forty university students were recruited through advertisements posted in the psychology department. Ten males and 10 females participated in each experiment. Ages ranged from 17 to 33 in Experiment 1 and 18 to 23 in Experiment 2. All students were paid for their participation.

\section{Design and Procedure}

Each student was told that the experimenter was interested in the way people form associations between words. He/she was then given a deck of 64 index cards with one word typed on each card. The words were common words of one or two syllables selected at random from a pool of nouns, verbs, and adjectives. The words were to be placed in piles, so that all of the words in a group had some kind of association. The subject sorted the words one at a time and was not permitted to remove a card once it had been placed in a group. The subjects were permitted as many sorting trials as they wished and were asked to create between 15 and 20 groups with three to five words per category. The cards were arranged so that all of the words in a pile were visible. Four different lists of words were used, and 5 subjects were tested on each list in each experiment.

After the student had achieved a satisfactory grouping of the words, he/she worked on a set of anagrams for $5 \mathrm{~min}$. During this time, the experimenter recorded the categories used by the subject and wrote the first item in each category on the response sheet for the cued recall test. In Experiment 1, the subjects took the free recall test, then worked on anagrams for another $5 \mathrm{~min}$, and then took the cued recall test. The same procedure was used in Experiment 2, except that the cued recall test came first. After the subjects finished the second recall test, they were asked to indicate on a list of strategies which they had used to group the words. They were also asked whether or not they had expected a recall test.

\section{RESULTS AND DISCUSSION}

All the subjects except 1 obeyed the sorting instructions; that person used only 14 categories, but included three to five items in each category. The data from this subject were included in all analyses. The mean total time spent in sorting the words into groups was $32.5 \mathrm{~min}(S D=12.7)$ in Experiment 1 and $29.8 \mathrm{~min}(S D=14.0)$ in Experiment 2 . In Experiment 1, 3 subjects required only one sorting trial, 14 used two sorting trials, 2 needed three trials, and 1 sorted the words four times. In Experiment 2, 3 subjects required only one sorting trial, 9 needed two trials, and 7 wanted three trials. (The time for each sorting trial and the number of sorting trials were not recorded 
Table 1

Numbers of Subjects Using Various Organizational Strategies

\begin{tabular}{lcccccc}
\hline & \multicolumn{6}{c}{ Strategy } \\
\cline { 2 - 7 } & Sentence & Imagery & Story & Alphabetic & Grammatical & Other \\
\hline Experiment 1 & 8 & 12 & 6 & 4 & 2 & 8 \\
Experiment 2 & 5 & 12 & 4 & 3 & 3 & 12 \\
Total & 13 & 24 & 10 & 7 & 5 & 20 \\
\hline
\end{tabular}

for 1 subject in Experiment 2.) The sorting strategies reported by subjects are shown in Table 1 . The most frequently reported strategy was the use of visual imagery to link the words of a group. The next most frequent strategy was the category "other," which was checked by half the subjects. These subjects further indicated the nature of the strategy used, which, in most cases, was a thematic grouping of some kind. In Experiment 1, 6 subjects said they expected a recall test: 5 of the 6 recalled more words in the cued recall test. In Experiment 2, 3 subjects said they expected a recall test: 1 recalled more words in cued recall, and 2 recalled fewer words.

For both free and cued recall tests, the dependent variable was the proportion of target items recalled. The target items were all the list words except those that were used as cues in the cued recall test. In cued recall, an item was considered to have been recalled if it was written on the response sheet, whether or not it was associated with the appropriate cue word. In Experiment 1, in which the free recall test came first, $78 \%$ of the target items were produced in the free recall test and $86 \%$ in the cued recall test $[t(19)=5.19, p<.001]$. Of the 20 subjects, only 1 produced more items in the free recall test, 2 showed no difference, and 17 produced more words in the cued recall test $(p<.001$ by a sign test). In Experiment 2 , $80 \%$ of the target words were recalled in the cued recall test and $74 \%$ in the free recall test $[t(19)=2.95$, $p<.01]$. Of the 20 subjects, 7 did better on the free recall test, 3 showed no difference, and the remaining 10 had higher recall on the cued recall test $(p>.10$ by the sign test).

The results demonstrate a beneficial effect of intralist cues in recall when the cues are chosen so that one retrieval cue represents each subjective category. In Experiment 1 , recall significantly improved from the first (free) recall test to the second (cued) recall test, whereas in Experiment 2, recall decreased from the cued test to the following free recall test. The magnitude of the cuing effect was smaller in Experiment 2 than in Experiment 1 and was not found for as many subjects, a finding that suggests that the order of tests may influence the cuing effect.

A further analysis was conducted of the number of categories recalled. In both free and cued recall, a category was considered to be recalled if at least one item other than the cue word was recalled from the category. In cued recall, the target items did not have to be written next to the cue in order for the category to be counted as having been recalled. Of the 17 subjects in Experi- ment 1 who recalled more words in the cued recall test than in free recall, 15 recalled more categories in cued recall ( $p=.002$ by a sign test). The mean numbers of categories recalled by these subjects were 14.8 and 16.8 for free and cued recall tests, respectively $[t(16)=5.22$, $p<.001$ ]. (If a strict scoring criterion is used in cued recall, so that a subjective category is considered to be recalled only if at least one target word is written beside the appropriate cue, the mean number of categories recalled decreases to 16.6.) In Experiment 2, only 10 subjects had higher recall on the cued recall test; all 10 of these subjects recalled more categories in cued than in free recall ( $p=.002$ by a sign test). For these 10 subjects, the mean number of categories recalled was 13.3 for free recall and 16.0 for cued recall $[t(9)=6.38$, $p<.001]$. (There were four instances in the free recall test in which a subject recalled only the cue word from a particular category. If these cases are included as categories recalled, the mean number of categories recalled in free recall rises to 13.7.) It is clear that the benefit of the cues is due to the increased number of subjective categories retrieved when cues are present.

An analysis of size of recalled categories was also undertaken for all subjects. In cued recall a lenient scoring criterion was used, according to which a subjective category was considered to have been recalled as long as at least one target item from the category was reported; the target item did not have to be written next to the cue word. In Experiment 1, the mean number of words recalled per category was 2.45 for free recall and 2.47 for cued recall $[t(19)=.503, p>.05]$. In Experiment 2, the mean category size was 2.32 in free recall and 2.33 in cued recall $[t(19)=.191, p>.05]$. There is no evidence at all that the presence of cues affects the number of items recalled from a category.

An analysis of category recall was also carried out on the data of those subjects who showed no improvement in recall when cues were supplied. In Experiment 1, only 3 of the 20 subjects fell into this category. Of the 3 , 1 subject recalled at least one word from every subjective category in free recall; the 2 remaining subjects recalled words from all categories except one. In Experiment 2, 10 subjects showed either no difference in performance in free and cued recall tests or lower recall when cues were present. Eight of these subjects recalled at least one word from every subjective category in free recall. The 2 remaining subjects had one instance each in which the only word recalled from a subjective category was the cue word. If these cases are included as examples of 
recalled categories, then all 10 subjects would be considered to have recalled all of the subjective units. The ineffectiveness of the retrieval cues, where it was observed, can clearly be attributed to the high level of category recall in the free recall test. There simply were no subjective units that were not retrieved in free recall but that were potentially retrievable given a suitable retrieval cue. Under these conditions, retrieval cues cannot increase recall.

The prediction of a beneficial effect of using list items as retrieval cues in recall of unrelated words rests on certain assumptions: that subjects organize the list into subjective units of at least two items, that some of these units are not recalled in a free recall test, and that the items chosen as retrieval cues are strongly associated with the remaining items in the unrecalled subjective units. In the present experiments, a sorting task was used to encourage organization of unrelated words into discrete subjective units, and retrieval cues were selected so that each subjective unit was represented. Under these conditions, increased recall of target items was observed in the presence of cues, and the improved recall was attributable to an increased number of subjective categories recalled. Nearly all of the subjects who showed no benefit from cues were observed to recall at least one item from each of the subjective categories they had created. These subjects had no unrecalled chunks, retrieval of which could be achieved by means of appropriate cues. The results indicate that under appropriate conditions, beneficial effects of part- list cuing can be observed. It appears that earlier studies reporting either no beneficial effects or negative effects of intralist cues were not performed under optimal conditions for effective cuing.

\section{REFERENCES}

Dong, T., \& KiNTsCH, W. (1968). Subjective retrieval cues in free recall. Journal of Verbal Learning \& Verbal Behavior, 7, 813-816.

MANDleR, G. (1967). Organization and memory. In K. W. Spence \& J. T. Spence (Eds.), The psychology of learning and motivation (Vol. 1, pp. 327-372). New York: Academic Press.

Mandler, G., \& Pearlstone, Z. (1966). Free and constrained concept learning and subsequent recall. Journal of Verbal Learning \& Verbal Behavior, 5, 126-131.

NiCKerSON, R. S. (1984). Retrieval inhibition from part-set cuing: A persisting enigma in memory research. Memory \& Cognition, 12, 531-552.

Reardon, E., Polzella, D., \& Brown, T. V. (1975). Re-examination of trace storage in free recall. Perceptual \& Motor Skills, 41, 383-393.

SlameckA, N. J. (1968). An examination of trace storage in free recall. Journal of Experimental Psychology, 76, 504-513.

SlameCKA, N. J. (1969). Testing for associative storage in multitrial free recall. Journal of Experimental Psychology, 81, 537-560.

Tulving, E., \& Pearlstone, Z. (1966). Availability versus accessibility of information in memory for words. Journal of Verbal Learning \& Verbal Behavior, 5, 381-391.

Wood, G. (1969). Retrieval cues and the accessibility of higher-order memory units in multitrial free recall. Journal of Verbal Learning \& Verbal Behavior, 8, 782-789.

(Manuscript received for publication November 10, 1987.) 